Liliana Andolpho Magalhães Guimarães ${ }^{a}$ (iD) https://orcid.org/0000-0002-9355-4015

Alessandra Laudelino Neto iD https://orcid.org/0000-0003-1918-2046

João Massuda Júnior ${ }^{\mathrm{a}}$ (D) https://orcid.org/0000-0003-0523-6285

aUniversidade Católica Dom Bosco, Programa de Mestrado e Doutorado em Psicologia. Campo Grande, MS, Brasil.

Contato:

Alessandra Laudelino Neto

E-mail:

alessandraneto.psico@gmail.com

Os autores declaram que o trabalho não foi subvencionado e que não há conflitos de interesses.

Os autores informam que o trabalho não foi apresentado em evento científico e que não foi baseado em dissertação ou tese.

\section{Intervenção integrada em saúde mental do trabalhador em uma corporação policial de Campo Grande (MS)}

\author{
Workers' mental health integrated intervention in a \\ police corporation of Campo Grande, MS, Brazil
}

\section{Resumo}

Introdução: transtornos mentais relacionados ao trabalho têm gerado crescente impacto econômico e social. A Organização Mundial da Saúde recomenda intervenções integradas no local de trabalho para lidar com os fatores que repercutem na saúde mental, visando a proposição de medidas preventivas mais efetivas. Objetivos: descrever e analisar uma intervenção integrada em saúde mental do trabalhador. Métodos: estudo descritivo-analítico de experiência profissional de atendimento psicológico em programa integrado de saúde mental do trabalhador em uma corporação de policiais rodoviários federais, em Campo Grande (MS). Resultados: o trabalho desenvolvido pode ter contribuído para reduzir o número de afastamentos por transtorno mental e os dias de afastamento do trabalho. Conclusão: a atuação integrada do serviço de atendimento ao trabalhador propiciou um momento de escuta, aprendizado e pertencimento que possivelmente repercutiu na redução do absenteísmo por doença.

Palavras-chave: saúde mental; planos e programas de saúde; polícia; saúde do trabalhador.

\begin{abstract}
Introduction: work-related mental illnesses have increasingly caused social and economical impact. The World Health Organization recommends integrated intervention aiming at implementig more effective prevention measures at workplace. Objective: to describe and analyze an integrated intervention in workers' mental health. Methods: descriptive/analytical study on an experience of psychological treatment administered in a workers' mental health integrated program held within a federal highway police corporation, in Campo Grande, MS, Brazil. Results: the measures adopted contributed to reduce sick leaves due to mental/behavioral disorders as well as the number of lost work days. Conclusion: the integrated measures offered to the workers a chance to learn, to be involved and listened, which possibly led to reduction in sick leaves.
\end{abstract}

Keywords: mental health; healthcare programs; police; occupational health. 


\section{Introdução}

As questões associadas à saúde mental têm assumido grande importância nas discussões sobre saúde pública em todo o mundo ${ }^{1}$. Um número substancial de pesquisas tem evidenciado, ao longo das últimas décadas, relações entre as condições psicossociais de trabalho e a saúde mental dos trabalhadores ${ }^{2-6}$. A União Europeia estimou que o custo econômico dos transtornos mentais relacionados ao trabalho para a economia de seus países-membros era da ordem de 3 a 4\% de seu Produto Interno Bruto (PIB), não sendo incluído neste cálculo os custos sociais, devido a sua complexa apuração $0^{7,8}$.

No Brasil, os transtornos mentais e do comportamento representam a terceira maior causa de ausências e incapacidades para o trabalho ${ }^{9}$. Em 2016, cerca de 127 mil trabalhadores brasileiros se afastaram por motivo de doença ou foram aposentados por invalidez devido a este tipo de transtorno ${ }^{9}$. O cenário impacta a realidade dos trabalhadores, suas famílias, empregadores, sistemas de saúde e sociedade em geral ${ }^{9}$.

Estudos ${ }^{6,10-14}$ relatam que a maioria dos trabalhadores que experimentam transtornos mentais comuns demoram a buscar atendimento especializado ou, muitas vezes, não o procuram. Esta demora para ter acesso a um tratamento adequado tende a ocasionar problemas no ambiente familiar, pessoal e profissional, por vezes a longo prazo, culminando com o absenteísmo e o presenteísmo laboral.

Embora diversas profissões sofram com estes transtornos, é notável que algumas atividades de trabalho são mais expostas, como as vinculadas às organizações de emergência (policiais, bombeiros, equipes de emergência médica, entre outras) ${ }^{15,16}$, que trabalham na perspectiva do socorro ou da segurança. A atividade profissional exercida pelo policial rodoviário federal é uma das mais estressantes e perigosas de acordo com a literatura nacional e internacional ${ }^{17-19}$, em decorrência do contato com acidentes graves, risco constante de morte, violência, ameaças à integridade física e psicológica, pressões ambientais e sociais, entre outros riscos. Tal cenário caracteriza os trabalhadores destas organizações como um grupo exposto a um alto risco psicossocial, podendo resultar em efeitos psicológicos, físicos e sociais negativos, como estresse relacionado com o trabalho, esgotamento ou depressão ${ }^{16}$. É imperativo, portanto, desenvolver ações com foco na promoção e prevenção em saúde mental no ambiente de trabalho desses profissionais.
Evidências científicas revelam a relação entre vivência diuturna do estresse, exposição a eventos adversos, desgaste resultante das atividades exercidas e maior suscetibilidade a cefaleias, dores musculoesqueléticas, alterações no sono, síndrome de burnout e transtorno por estresse pós-traumático, além de maior probabilidade de acidentes relacionados ao trabalho, inclusive fatais ${ }^{15}$.

Uma forma de combater os malefícios deste contexto ocupacional é desenvolver intervenções que integrem ações em três níveis ${ }^{5,17,20}$ : (i) prevenção primária, voltada a fontes estressoras associadas ao indivíduo e aos sistemas sociais; (ii) prevenção secundária, visando minimizar os impactos e promover recursos individuais e sociais; e (iii) prevenção terciária, com o intuito de reabilitar os indivíduos e os sistemas sociais, como o contexto do trabalho ${ }^{15}$. Segundo Lamontagne et al. ${ }^{5}$ :

\begin{abstract}
Algumas estratégias de intervenção podem ser classificadas de maneiras diferentes (por exemplo, aumentar a capacidade de resiliência ou capacidade de enfrentamento dos trabalhadores pode ser considerada prevenção primária se for feita antes de um problema de saúde mental ter ocorrido, e secundária se impedir a progressão de um problema existente). (p. 3, tradução livre)
\end{abstract}

A Organização Mundial da Saúde preconiza uma abordagem integrada para examinar todos os aspectos do problema, de modo a identificar uma ampla gama de soluções eficazes para a realidade em questão ${ }^{21}$. Este estudo teve como objetivo descrever e analisar uma prática de intervenção em saúde mental com abordagem integrada, voltada a policiais rodoviários federais.

\section{Percurso metodológico}

Trata-se de um estudo descritivo-analítico de experiência profissional de atendimento psicológico em um programa de saúde mental do trabalhador, com abordagem integrada, desenvolvido em 2016 em uma organização de emergência, no caso uma corporação policial atuante no município de Campo Grande (MS).

A abordagem integrada adotada como referencial foi a preconizada pela Organização Mundial da Saúde no documento Healthy workplaces: a model for action for employers, workers, policy-makers and practitioners $^{21}$, que amparou este estudo em três aspectos: (i) intervenção individual, com ações relacionadas à psicoeducação ${ }^{5} \mathrm{e}$ à gestão dos primeiros socorros psicológicos (plantão psicológico), além de um sistema de atenção ao trabalhador, enfoque 
deste relato; (ii) intervenção grupal, centrada nos sistemas sociais (e.g., treinamentos, palestras, grupos); e (iii) intervenção organizacional, voltada ao ambiente de trabalho (e.g., consultorias relacionadas ao cotidiano, organização e contexto do trabalho, entre outras).

As ações desenvolvidas foram baseadas no campo teórico da psicologia da saúde ocupacional, pelo viés do bem-estar em organizações de emergência ${ }^{15}$. Quanto às medidas de prevenção primária e secundária, priorizou-se a capacidade de gestão das emoções (e.g., hardiness, gestão do estresse, lócus de controle, percepção social e apoio nos denominados primeiros socorros psicológicos em nível individual) ${ }^{15}$. A prevenção terciária voltou-se para o apoio psicoterapêutico e a psicotraumatologia, priorizando a multidisciplinaridade e a abordagem biopsicossocial, conforme debatido na 11th Conference of the European Academy of Occupational Health Psychology, em 201422.

Todos os preceitos éticos foram respeitados, conforme aprovação por Comitê de Ética em Pesquisa, número do CAAE: 54473616.2.0000.5162, número do parecer: 1482980 .

\section{Resultados e discussão}

\section{A construção e implantação do atendimento}

Após a celebração do convênio entre a Universidade Católica Dom Bosco de Campo Grande (MS) e a Terceira Superintendência da Polícia Rodoviária Federal de Mato Grosso do Sul, foi iniciada a construção do projeto de Intervenção Integrada em Saúde Mental do Trabalhador, tendo como público-alvo inicial cerca de 250 servidores alocados na referida superintendência em Campo Grande, MS.

As seguintes linhas de ação foram propostas inicialmente: (i) pesquisa diagnóstica, com levantamento epidemiológico e de demandas, desenvolvida no Programa de Pós-Graduação em Psicologia da Universidade Católica Dom Bosco (e.g. diagnósticos micro, meso e macro por meio de instrumentos, obtidos individualmente e para o grupo); (ii) pré-implementação; (iii) plantão psicológico in loco, com foco no acolhimento profissional e na compreensão da demanda, colhendo informações, direcionando tratamentos e reduzindo o estigma; (iv) atendimento psicoterapêutico, realizado no Ambulatório de Saúde Mental do Trabalhador, criado para este projeto, que funciona na clínica-escola de psicologia da referida universidade; e (v) ações psicoeducativas (e.g., consultoria sobre problemas relacionais do cotidiano, da organização e do contexto de trabalho, palestras informativas, grupos de conversa e treinamento de habilidades voltados à identidade de equipe, à coesão de grupo e à definição de papéis).

A implantação do serviço de atendimento integrado contou com uma equipe multidisciplinar formada por uma psicóloga docente-pesquisadora (coordenadora geral do projeto); mestrandos, doutorandos e pós-doutorandos de diferentes áreas de atuação (e.g., administrador, assistente social, enfermeiro e psicólogos) vinculados ao programa de pós-graduação stricto sensu em Psicologia da Saúde; e acadêmicos do estágio específico em Saúde na Contemporaneidade do curso de graduação em psicologia.

Na fase de pré-implementação, houve a sistematização dos processos de intervenção com o objetivo de sensibilizar os envolvidos e possibilitar um atendimento com equidade, pautado na ética, proteção de direitos, bem-estar e dignidade dos participantes. Nesta etapa, foi criado um protocolo de atendimento que normatizou os processos, o fluxo de informações e a sequência operacional. Foram disponibilizados ainda orientações, ferramentas e instrumentos técnicos considerados necessários para a prestação de um serviço qualificado por parte da equipe de trabalho.

Para melhor esclarecer o processo de atendimento em contexto ocupacional, foram elaborados fluxogramas (Figuras 1 e 2). As prioridades de atendimento também foram categorizadas, assumindo a seguinte estrutura: Prioridade 1 - atendimento de emergência (quando há uma situação que não pode ser adiada); Prioridade 2 - atendimento de urgência (quando há uma situação crítica ou algo iminente); e Prioridade 3 - atendimento eletivo (não apresenta as características anteriores). Tais prioridades se referem tanto à gravidade da situação analisada (e.g., tentativa de suicídio, transtorno de ansiedade generalizada, transtorno de estresse pós-traumático) quanto à percepção da gravidade do evento pelo trabalhador.

O passo subsequente para implementar o projeto aqui descrito se deu no ambiente de trabalho dos policiais rodoviários federais, com um serviço de plantão psicológico na sede da corporação. Os atendimentos realizados nesse local ocorreram pela perspectiva de uma prática clínica contemporânea, comprometida com a escuta e o acolhimento do outro e apoiada em uma reflexão ética e política. 


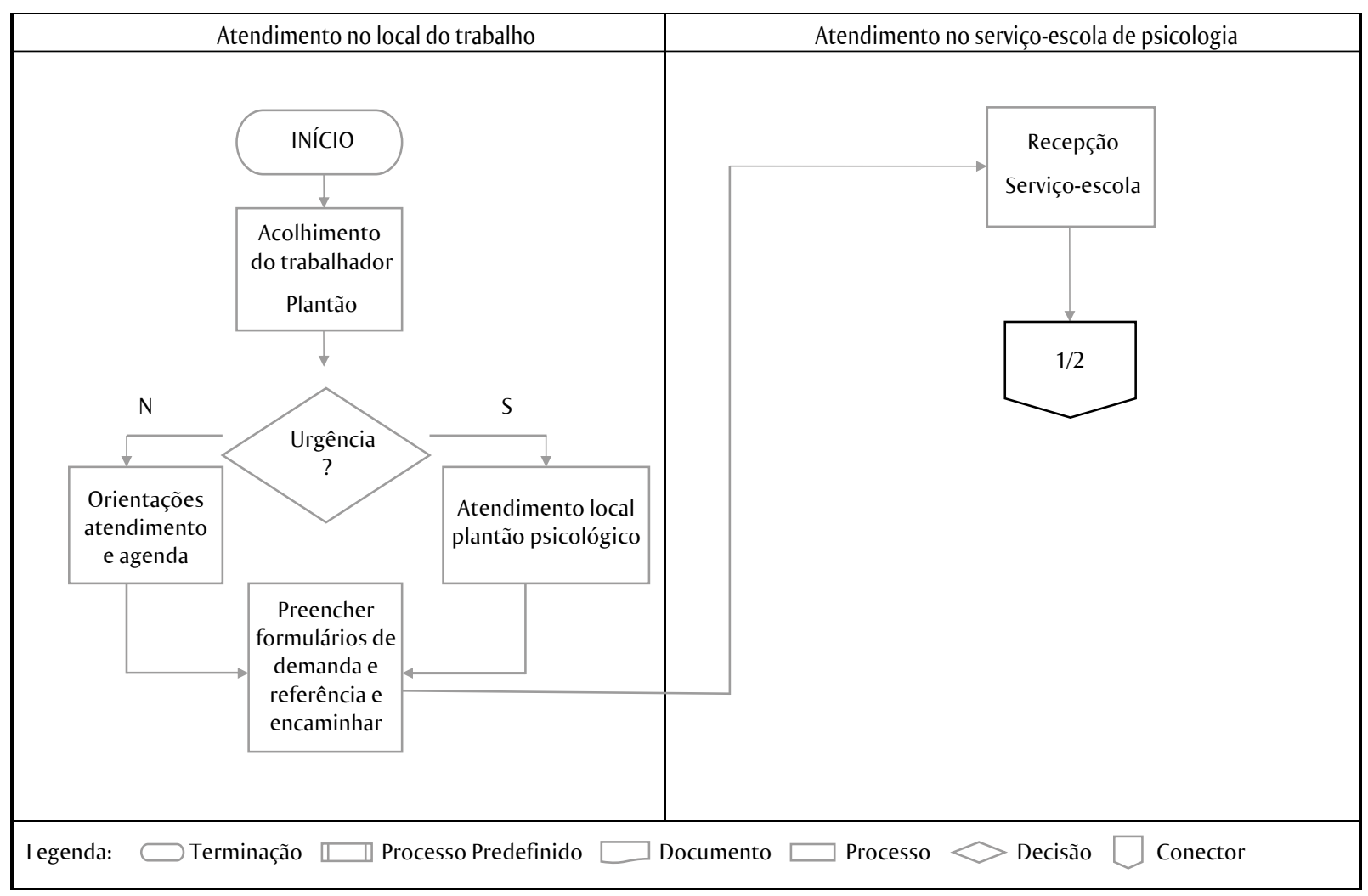

Figura 1 Ambulatório de Saúde Mental do Trabalhador: fluxograma de atendimento no local do trabalho (01/02)

O atendimento prestado pela equipe envolvida no projeto buscava proporcionar ao indivíduo que procurava o serviço um momento em que se sentisse ouvido. Tal conduta favorecia possíveis ressignificações e servia de base para que o indivíduo aguçasse sua percepção acerca de questões-problema relacionadas a suas vivências e a seu trabalho ${ }^{17,23}$.

É importante destacar que o plantão psicológico, no formato proposto neste projeto, é reconhecido pelo Conselho Federal de Psicologia. O programa buscou lidar com as queixas dos usuários enquanto sintomas de uma demanda e, nesse sentido, teve a função de iniciar um processo de autorreorganização, esclarecendo uma problemática e se constituindo como espaço de expressão e acolhimento de angústias. O serviço pôde ainda atuar na diminuição da ansiedade e da angústia, possibilitando o surgimento de recursos inerentes à pessoa para que ela busque soluções para seu impasse ${ }^{23}$.

Mediante a análise das prioridades e do interesse do trabalhador, o plantonista tinha a possibilidade de fazer encaminhamentos após o atendimento no plantão. Uma das alternativas possíveis, quando necessário, era o encaminhamento para atendimento na clínica-escola de psicologia (Figura 2).

Uma vez encaminhado à clínica-escola de psicologia, o indivíduo devia passar por uma triagem que visava garantir o alinhamento do atendimento a suas necessidades. Durante esse processo, buscou-se compreender as demandas do trabalhador (e.g. atendimento individual ou grupal) e quais seriam as melhores condutas e procedimentos terapêuticos e propedêuticos a se adotar. Para isso, foram preenchidos formulários sobre o perfil profissiográfico (documento histórico-laboral do trabalhador desenvolvido especialmente para essa população). Nessa fase, também foi apresentado o termo de consentimento livre e esclarecido de adesão ao serviço ofertado pela clínica-escola, contendo, em linguagem clara e objetiva, todas as informações do projeto. 


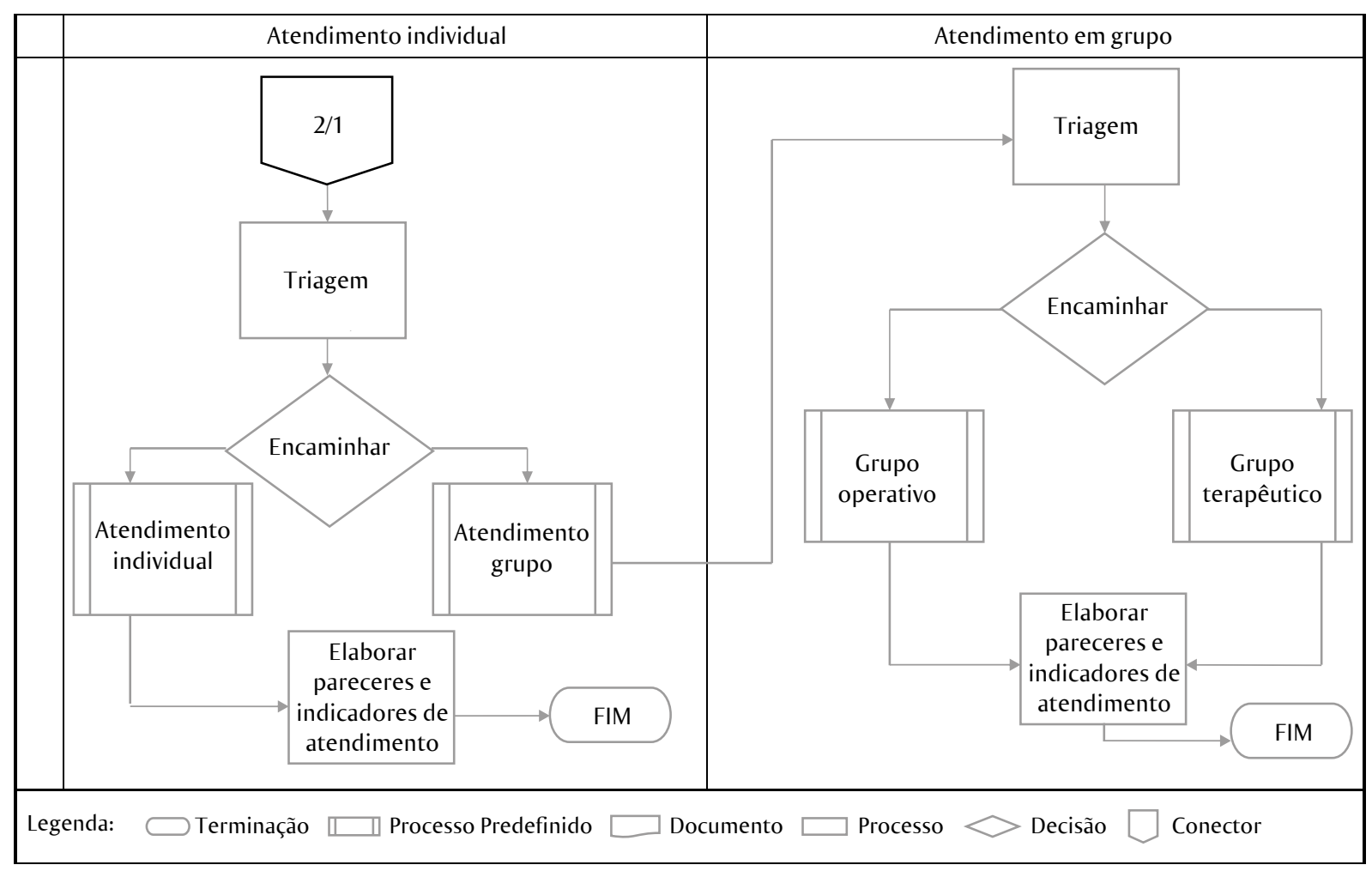

Figura 2 Ambulatório de Saúde Mental do Trabalhador: fluxograma de atendimento na Clínica-Escola (02/02)

\section{Desenvolvimento do trabalho}

Durante o ano de 2016, foram realizados 52 atendimentos no plantão psicológico nas instalações da Polícia Rodoviária Federal e 109 atendimentos no ambulatório da clínica-escola da universidade. Participaram do projeto 41 servidores da polícia, a maioria do sexo masculino $(63,4 \%)$, com idade média e desvio-padrão de 38,5 e 12,4 anos, respectivamente. Os atendimentos foram classificados como emergentes, urgentes e eletivos. De acordo com a análise dos prontuários registrados, 58,5\% dos atendimentos foram identificados como urgentes (e.g., alto nível de estresse, uso prolongado de álcool); 29,3\% como eletivos (e.g., estresse, ansiedade); e 12,2\% como emergentes (e.g., ideações e tentativas suicidas, transtornos depressivos e ansiosos, transtorno por estresse pós-traumático). Por diferentes razões laborais e ocorrências de ordem macro, que promoviam o deslocamento massivo dos policiais, não foi possível efetivar atendimento grupal no período pesquisado.

Os casos relatados foram diversos e tratavam de questões relacionadas ao trabalho, família, relacionamento com colegas, amigos e familiares, consumo excessivo de medicamentos e álcool, obesidade e sexualidade, entre outras ${ }^{b}$. Os serviços oferecidos contaram com a participação espontânea dos trabalhadores de forma gradativa, sendo o ambiente físico e experiencial do atendimento em plantão psicológico criado conjuntamente com os policiais, o que propiciou maior adesão e confiabilidade.

A cada semana, um trabalhador integrava elementos a essa vivência, tais como disponibilizar cadeiras para montar uma sala de espera, imprimir um cartaz com o dizer "em atendimento" para ser fixado na porta da sala e sugerir o atendimento em outros horários e unidades, entre outras colaborações. Dessa maneira, ao perceber o serviço como seu, o trabalhador estabeleceu um vínculo fundamental de pertencimento, segurança e apoio.

Em consonância, um estudo de follow up, realizado por cerca de 3 anos (2002-2005) com 1.867 participantes em unidades de emergência de oito hospitais em cinco locais culturalmente diferentes (Campinas, Brasil; Chennai, Índia; Colombo, Sri Lanka; Karaj, República Islâmica do Irã; e Yuncheng, China) concluiu que programas de prevenção contendo intervenções breves, semelhantes ao plantão psicológico, atuam como uma rede temporária de apoio e são eficazes para conscientizar os trabalhadores e encontrar formas de solucionar problemas complexos, como ideações suicidas ou mesmo suicídio ${ }^{24}$.

b Informações confidenciais dos prontuários foram preservadas. 
O acompanhamento psicoterapêutico individual na clínica-escola de psicologia, mediante o encaminhamento via plantão psicológico, ocorreu semanalmente. Os atendimentos foram efetuados por estagiários da graduação do último ano de psicologia, sob supervisão de psicólogos, mestrandos e doutorandos, integrantes do programa de pós-graduação stricto sensu em Psicologia da Saúde da Universidade, e orientação da coordenadora do projeto.

Este formato de intervenção - utilizando-se de discussões teórico-reflexivas com uma equipe multidisciplinar e atuação prática, tanto in loco, na sede da Polícia Rodoviária Federal, quanto na clínica-escola de psicologia - mostrou-se eficaz no atendimento às necessidades dos servidores e permitiu que se desenvolvesse um raciocínio clínico ampliado, por meio da reflexão ética sobre a prática psicológica.

A adesão crescente ao longo do desenvolvimento do projeto denota que as ações tiveram um impacto positivo para o ambiente de trabalho e para os servidores atendidos. No entanto, avaliar a extensão deste impacto é uma tarefa extremamente complexa, uma vez que não é possível isolar o efeito sobre a saúde mental dos trabalhadores de outras ações e mudanças no ambiente de trabalho.

Apesar das dificuldades para mensurar os efeitos do programa desenvolvido, há um indicativo da eficácia da intervenção: a redução significativa dos dias e casos de afastamentos associados ao CID-F (CID-10 - Classificação Internacional de Doenças/Grupo F - Transtornos Mentais e do Comportamento) um ano após a implantação do programa, segundo dados fornecidos pela Polícia Rodoviária Federal mediante o Subsistema Integrado de Atenção à Saúde do Servidor.

Em 2015, por exemplo, os transtornos mentais e do comportamento - como transtorno afetivo bipolar e episódios depressivos, entre outros (CIDs-10 ${ }^{\mathrm{C}}$ F14, F315, F321, F322, F330, F331, F332 e F412) constavam na lista das 20 maiores causas de afastamento e representaram $28,8 \%$ das ausências no trabalho em decorrência de problemas de saúde. Já no ano de 2016 houve uma redução, sendo que apenas os CIDs-10 ${ }^{\mathrm{d}}$ F31, F330, F332 e F412 estavam na lista das 20 maiores causas de afastamento do trabalho. Essas ocorrências representaram $14,1 \%$ das faltas em razão de problemas de saúde.

Analisando os números absolutos, a redução é ainda mais significativa, pois em 2015 foram registrados 32 pedidos de afastamentos por transtornos mentais e do comportamento do grupo F da CID-10, enquanto em 2016 registraram-se apenas 15. Quanto aos dias de afastamento do trabalho, em 2015 foram 1.062 dias, enquanto em 2016 registraram-se 477 dias de ausência ligadas ao grupo F da CID-10.

Os transtornos mentais decorrentes da incapacitação para atividades ocupacionais ocasionam grande impacto sobre os indivíduos, famílias e comunidades, e têm aumentado, como apontam estatísticas oficiais nacionais e internacionais ${ }^{7,21}$. A atuação integrada do serviço de atendimento ao trabalhador propiciou um momento de escuta, aprendizado e pertencimento que possivelmente repercutiu na redução do absenteísmo por doença.

Um estudo realizado com 2.481 trabalhadores australianos com o objetivo de conhecer padrões longitudinais de absenteísmo em tempo integral, apontou que funcionários com alto nível de absenteísmo apresentavam mais dor e menos saúde física e mental quando comparados àqueles com absenteísmo em nível moderado, sugerindo que altas taxas de absenteísmo podem refletir fatores de restrição de saúde ${ }^{25}$. Outro estudo de intervenção implementado em diversos países é o Mental Health First Aid (MHFA)e, que atua para melhorar a conscientização em saúde mental e desenvolver habilidades e conhecimentos no gerenciamento destes problemas ${ }^{5}$.

No presente estudo, foram realizadas também ações psicoeducativas, como roda de conversa nos setores com orientações para prevenção do estresse e ansiedade, palestras psicoeducativas com gestores e um treinamento sobre desenvolvimento das relações humanas que visou fortalecer os vínculos e as relações inter e intraorganizacionais (nos níveis micro e meso).

Para a saúde do trabalhador, essas ações tiveram o propósito de ampliar a compreensão sobre o papel do trabalho no processo saúde-doença, além de esclarecer e contribuir para uma lógica preventiva e de promoção da saúde. Essa é considerada uma

c Respectivamente: F14 - Transtornos mentais e comportamentais devido ao uso da cocaína; F315 - Transtorno afetivo bipolar, episódio atual depressivo grave com sintomas psicóticos; F321 - Episódio depressivo moderado; F322 - Episódio depressivo grave sem sintomas psicóticos; F330 - Transtorno depressivo recorrente, episódio atual leve; F331 - Transtorno depressivo recorrente, episódio atual moderado; F332 - Transtorno depressivo recorrente, episódio atual grave sem sintomas psicóticos; e F412 - Transtorno misto ansioso e depressivo.

d Respectivamente: F31 - Transtorno afetivo bipolar; F330 - Transtorno depressivo recorrente, episódio atual leve; F332 Transtorno depressivo recorrente, episódio atual grave sem sintomas psicóticos; e F412 - Transtorno misto ansioso e depressivo.

e Mental Health First Aid USA é gerenciado, operado e disseminado pela National Council for Behavioral Health e o Missouri Department of Mental Health (https://www.mentalhealthfirstaid.org/). 
área-chave de intervenção, conforme o Programa de Trabalho Psicologicamente Saudável proposto pela American Psychological Association (APA) ${ }^{26}$, que contemplou os níveis micro, meso e macro organizacionais, estabelecendo que o cenário social, político e do trabalho deve ampliar as possibilidades de atuação e intervenção, com uma visão de ser humano inserido socialmente ${ }^{27}$.

Em próximos estudos, deverão ser aplicados instrumentos antes e depois das intervenções (estudos de validação clínica) para verificar a eficácia e a efetividade da intervenção realizada. Também deverão ser controladas algumas variáveis - por exemplo, se o policial rodoviário desenvolve suas atividades em pista ou na parte administrativa, para maior acurácia dos resultados.

Como reconhecimento do trabalho com a Terceira Superintendência da Polícia Rodoviária Federal de Mato Grosso do Sul, a Universidade Católica Dom Bosco recebeu da corporação uma homenagem oficial pela excelência da intervenção em benefício da saúde mental dos trabalhadores, destacando a possibilidade de replicação dessa experiência em âmbito nacional, em todas as unidades da Polícia Rodoviária Federal. Também a Câmara Municipal de Campo Grande contemplou a equipe, na figura da coordenadora do projeto, com diploma de congratulações por serviços prestados à corporação e à população.

\section{Considerações finais}

Este estudo buscou oferecer elementos para uma reflexão sobre os benefícios de um serviço de intervenção integrada em saúde mental do trabalhador e suas repercussões no ambiente laboral, sobretudo em profissões que envolvem grande sobrecarga física e emocional, propondo uma lógica preventiva e de promoção da saúde. Por mais que os resultados apresentados não possam ser inteiramente creditados às iniciativas apresentadas neste trabalho, é inegável que as medidas aplicadas impactaram significativamente em indicadores institucionais associados à saúde dos servidores.

Como limitação, pode-se dizer que não foi possível um estudo de validação clínica antes e depois da intervenção. Por fim, destaca-se que para atender a diversidade e singularidade das demandas no contexto do trabalho, necessita-se do apoio de políticas institucionais e públicas mais amplas relacionadas à saúde do trabalhador, para integrar prática, política e pesquisa.

\section{Contribuição de autoria}

Os autores contribuíram igualmente nas diversas fases da investigação e da redação do artigo, que incluíram coleta de dados, análise, redação e revisão do texto, e assumem responsabilidade pública pelo seu conteúdo.

\section{Referências}

1. Kessler RC, Üstün TB, editores. The WHO world mental health surveys: global perspectives on the epidemiology of mental disorders. Geneva: WHO; 2008.

2. Guimarães LAM, Grubits S. Série saúde mental e trabalho. São Paulo: Casa do Psicólogo; 2004.

3. Kessler RC, Angermeyer M, Anthony JC, De Graaf $\mathrm{R}$, Demyttenaere K, Gasquet I, et al. Lifetime prevalence and age-of-onset distributions of mental disorders in the World Health Organization's World Mental Health Survey Initiative. World Psychiatry. 2007;6(3):168-76.

4. LaMontagne AD, Sanderson K, Cocker F. Estimating the economic benefits of eliminating job strain as a risk factor for depression [Internet]. Melbourne: Victorian Heath Promotion Foundation, 2010 [citado em 19 jun 2020]. Disponível em: https://www.vichealth.vic.gov.au/jobstrain

5. LaMontagne AD, Martin A, Page KM, Reavley NJ, Noblet AJ, Milner AJ, et al. Workplace mental health: developing a integrated intervention approach. BMC Psychiatry. 2014;14(131).
6. Schelvis RMC, Wiezer NM, van der Beek AJ, Twisk JWR, Bohlmeijer ET, Hengel KMO. The effect of an organizational level participatory intervention in secondary vocational education on work-related health outcomes: results of a controlled trial. BMC Public Health. 2017;17(141).

7. International Labour Office. Mental health in the workplace [Internet]. Geneva: International Labour Office; 2000 [citado em 19 jun 2020]. Disponível em: http://www.ilo.org/wcmsp5/groups/public/@ ed_emp/@ifp_skills/documents/publication/ wcms_108221.pdf

8. Organisation for Economic Co-Operation and Development. Sick on the job?: myths and realities about mental health and work. Paris: Organisation for Economic Co-Operation and Development; 2012 [citado em 19 jun 2020]. Disponível em: https:/www.gamian.eu/wpcontent/uploads/2014/05/Miranda_OECD_sick-onthe-job_presentation_Vilnius-2013.pdf

9. Brasil. Ministério da Fazenda. Adoecimento mental e trabalho: a concessão de benefícios por 
incapacidade relacionados a transtornos mentais e comportamentais entre 2012 e 2016: $1^{\circ}$ boletim quadrimestral sobre benefícios por incapacidade de 2017. Brasília: Ministério da Fazenda; 2017.

10. Bovopoulos N, Jorm AF, Bond KS, LaMontagne AD, Reavley NJ, Kelly CM, et al. Providing mental health first aid in the workplace: a Delphi consensus study. BMC Psychol. 2016;4(41).

11. de Graaf R, Tuithof M, van Dorsselaer S, ten Have M. Comparing the effects on work performance of mental and physical disorders. Soc Psychiatry Psychiatr Epidemiol. 2012;47(11)1873-83.

12. Hilton MF, Whiteford HA, Sheridan JS, Cleary CM, Chant DC, Wang PS, et al. The prevalence of psychological distress in employees and associated occupational risk factors. J Occup Environ Med. 2008;50(7):746-57.

13. Hargrove MB, Quick JC, Nelson DL, Quick JD. The theory of preventive stress management: a 33-year review and evaluation. Stress Health. 2011;27(3):182-93.

14. Kalimo R. Reversed causality: a need to revisit systems modeling of work-stress-health relationships. Scand J Work Environ Health. 2005;31(1):1-2.

15. Ângelo RP. Psicologia da saúde ocupacional em organizações de emergência. In: Chambel MJ, coordenadora. Psicologia da saúde ocupacional. Lisboa: Pactor; 2016. p. 113-134.

16. European Agency for Safety and Health at Work. Emergency services: a literature review on occupational safety and health risks. Milczarek M, editora. Luxembourg: Publications Office of the European Union; 2011.

17. Vasques-Menezes I, Fernandes SRP, Guimarães LAM, Lima EP. Saúde mental e trabalho: uma proposta de intervenção em contextos organizacionais. In: Mendonça $\mathrm{H}$, Ferreira MC, Neiva ER. Análise e diagnóstico organizacional: teoria e prática. São Paulo: Vetor; 2016. p. 251-72.

18. Felix YTM, Catão MF. Envelhecimento e aposentadoria por policiais rodoviários. Psicol Soc. 2013;25(2):420-9.
19. Laudelino Neto A, Gomes ECVV, Oliveira FF, Massuda Junior J, Rocha BC, Fagundes DF, et al. Comportamento suicida no contexto policial. In: Franco AA, organizadora. Pessoas, trabalho e organizações. Ituiutaba: Barlavento; 2017. p. 51-62.

20. Glina DMR, Rocha LE. Prevenção do estresse no trabalho. In: Glina DMR, Rocha LE, organizadores. Saúde mental no trabalho da teoria à prática. São Paulo: Roca; 2010. p. 113-153.

21. World Health Organization. Healthy workplaces: a model for action: for employers, workers, policymakers and practitioners. Geneva: WHO; 2010 [citado em 19 jun 2020]. Disponível em: http:// www.who.int/occupational health/publications/ healthy_workplaces_model.pdf

22. Andreou N, Jain A, Hollis D, Hassard J, Teoh K. Book of proceedings: 11th Conference of the European Academy of Occupational Health Psychology: "Looking at the Past - Planning for the Future: Capitalizing on OHP Multidisciplinarity". Nottingham: European Academy of Occupational Health Psychology; 2014.

23. Rebouças MSS, Dutra E. Plantão psicológico: uma prática clínica da contemporaneidade. Rev Abordagem Gestalt. 2010;16(1):19-28.

24. Fleischmann A, Bertolote JM, Wasserman D, De Leo D, Bolhari J, Botega NJ, et al. Effectiveness of brief intervention and contact for suicide attempters: a randomized controlled trial in five countries. Bull World Health Organ. 2008;86(9):703-9.

25. Magee CA,Caputi P, Lee JK. Distinct longitudinal patterns of absenteeism and their antecedents in full-time Australian employees. J Occup Health Psychol. 2016;21(1):24-36.

26. Grawitch MJ, Ballard DW. The psychologically healthy workplace: building a win-win environment for organizations and employees [Internet]. Washington: American Psychological Association; 2015.

27. Borges LO, Guimarães LAM, Silva SS. Diagnóstico e promoção da saúde psíquica do trabalho. In: Borges LO, Mourão L, organizadoras. O trabalho e as organizações: atuações a partir da psicologia. Porto Alegre: Artmed; 2013. p. 581-612 